\title{
STRONG LIMIT THEOREMS FOR ORTHOGONAL SEQUENCES IN VON NEUMANN ALGEBRAS
}

\author{
R. JAJTE ${ }^{1}$
}

\begin{abstract}
Let $A$ be a von Neumann algebra with a faithful normal state $\phi$. It is shown that if a sequence $\left(x_{n}\right)$ in $A$ is orthogonal relative to $\phi$ and satisfies the condition
\end{abstract}

$$
\sum_{h} \phi\left(\left|x_{h}\right|^{2}\right)\left(\frac{\log k}{k}\right)^{2}<\infty .
$$

then ${ }_{n}^{1} \sum_{h=1}^{n} x_{h} \rightarrow 0$ almost uniformly in $A$. Some other results related to this theorem are also discussed.

1. Preliminaries. Strong limit theorems in a von Neumann algebra with a faithful normal state, close to the classical ones, have been obtained for the ergodic averages and martingales (see, for example, [4-7]). To the best of our knowledge there are only very few results concerning sequences whose elements are orthogonal (or uncorrelated or independent) relative to a state: for uniformly bounded sequences [3, 8] and for sequences satisfying some rather special conditions of Rosenblatt's type [5]. Let us notice here that many results similar to the classical ones are known for traces, i.e. for finite von Neumann algebras $[3,8]$. However, for states the proofs are more complicated and, as a rule, need some new approach because the states, in general, are not subadditive on the lattice of projections in a von Neumann algebra. In case of a noncommutative von Neumann algebra, the notion of independence of its subalgebras with respect to a state [3] seems to be very restrictive, especially when the state is not tracial. On the other hand, some ideas of the correlation theory of stochastic processes can be developed in a natural way on the ground of quantum mechanics [1]. In particular, it seems to be important to have some information about the asymptotic behaviour of sequences of uncorrelated observables. This is the motivation of our work. The main goal of this paper is to prove a strong law of large numbers for sequences orthogonal relative to a state. Let us begin with some notation and definitions. Throughout, $A$ will denote a von Neumann algebra with a faithful normal state $\Phi$. For a projection $p$, always $p^{\perp}=1-p$, and $|x|^{2}=x^{*} x$ for

Received by the editors June 8, 1984 and, in revised form. August 16, 1984.

1980 Mathematics Subject Classification. Primary 46L10, 81C20, 60F15.

Kel words and phrases. von Neumann algebras, faithful normal state, orthogonal sequences, independent sequences, almost uniform convergence, almost complete convergence, strong law of large numbers.

'On leave from Lodz University, Poland. This paper was written while the author held a visiting position at the University of Tennessee, whose hospitality he gratefully acknowledges. 
every $x \in A$. We say that a sequence $\left(x_{n}\right)$ in $A$ converges almost uniformly to $x$ if, for each $\varepsilon>0$, there is a projection $p \in A$ with $\phi\left(p^{\perp}\right)<\varepsilon$ and such that

$$
\left\|\left(x_{n}-x\right) p\right\| \rightarrow 0 \text { as } n \rightarrow \infty .
$$

A sequence $\left(x_{n}\right)$ in $A$ is said to be convergent almost completely to $x$ if, for every $\varepsilon>0$, there exists a sequence $\left(q_{n}\right)$ of projections in $A$ such that $\Sigma_{n} \phi\left(1-q_{n}\right)<\infty$ and $\left\|\left(x_{n}-x\right) q_{n}\right\|<\varepsilon$ for $n=1,2, \ldots$. Relations between the almost complete and almost uniform convergence will be discussed in the Appendix.

Two elements $x$ and $y$ in $A$ are said to be orthogonal relative to $\phi$ if $\phi\left(x^{*} y\right)=0$. Two elements $x$ and $y$ are said to be uncorrelated (relative to $\phi$ ) if $\phi(x y)=\phi(x) \phi(y)$. Of course, both relations are symmetric (it is enough to take the adjoints).

Obviously, if $x$ and $y$ are uncorrelated, then the "centered" elements $x-\phi(x)$ and $y-\phi(y)$ are orthogonal. For a self adjoint operator $x$ in $A$ and a Borel set $Z$ on the real line, the symbol $e_{Z}(x)$ will denote the spectral projection of $x$ corresponding to the set $Z$; in particular,

$$
x=\int_{-\infty}^{\infty} \lambda e_{d \lambda}(x) \quad \text { (spectral representation). }
$$

In the sequel we shall need the following theorem which is a special case of the noncommutative maximal ergodic theorem of Goldstein [5].

THEOREM 1. Let $\left(a_{n}\right)$ be a sequence of positive elements in $A$ and let $\left(\varepsilon_{k}\right)$ be a sequence of positive numbers such that

$$
\sum_{k=1}^{\infty} \varepsilon_{k}^{-1} \phi\left(a_{k}\right)<\frac{1}{2} .
$$

Then there exists a projection $p \in A$ with $\phi(p) \geqslant 1-\sum_{k=1}^{\infty} \varepsilon_{k}^{-1} \phi\left(a_{k}\right)$ and such that $\left\|p a_{n} p\right\|<2 \varepsilon_{n}$ for $n=1,2, \ldots$.

2. Strong law of large numbers. In this section we prove the following strong limit theorem on sequences orthogonal relative to a state.

THEOREM 2. Let $A$ be a von Neumann algebra with a faithful normal state $\phi$ and let $\left(x_{n}\right)$ be a sequence of pairwise orthogonal elements of $A\left(i . e . \phi\left(x_{n}^{*} x_{m}\right)=0\right.$ for $\left.n \neq m\right)$. If

$$
\sum_{n=1}^{\infty}\left(\frac{\log n}{n}\right)^{2} \phi\left(\left|x_{n}\right|^{2}\right)<\infty
$$

then the averages

$$
S_{n}=\frac{1}{n} \sum_{k=1}^{n} x_{k}
$$

converge to zero almost uniformly.

In order to prove this theorem we start with the following

Proposition. Let $\left(y_{n}\right)$ be a sequence of pairwise orthogonal elements of $A$. Put

$$
t_{n}=\sum_{k=1}^{n} y_{k} \text {. }
$$


Then there exists in $A$ a sequence $\left(B_{m}\right)$ of positive operators such that

$$
\left|t_{n}\right|^{2} \leqslant(m+1) B_{m} \quad \text { for } 1 \leqslant n \leqslant 2^{m}
$$

and

$$
\phi\left(B_{m}\right) \leqslant(m+1) \sum_{k=1}^{2^{m}} \phi\left(\left|y_{k}\right|^{2}\right) .
$$

Proof. The proof is based on the idea which goes back to Plancherel [10] and is well known in the theory of orthogonal series [2]. Namely, we start from the dyadic representation of the index $n$. Divide the interval $I=\left(0,2^{m}\right]$ into intervals $\left(0,2^{m-1}\right]$ and $\left(2^{m-1}, 2^{m}\right]$, each of these intervals into halves, and so on; we obtain in this way a sequence of partitions of $I$. The elements of the first partition are of length $2^{m-1}$, the elements of the $r$ th partition are of length $2^{m-r}$. For a positive integer $n \leqslant 2^{m}$, we take its dyadic representaion. Then the interval $(0, n]$ can be written as the sum of at most $m$ disjoint intervals $I_{j}^{(n)}$, each of which belongs to a different partition, that is,

$$
(0, n]=\bigcup_{j=0}^{m} I_{j}^{(n)}
$$

where $I_{j}^{(n)}$ is empty or of length $\left|I_{j}^{(n)}\right|=2^{j}(j=1,2, \ldots, m)$. We can write

$$
t_{n}=\sum_{j=0}^{m} \sum_{k \in I_{j}^{(n)}} y_{k}
$$

(of course, we put $\sum_{k \in I_{1}^{(n)}} y_{k}=0$ in the case $I_{j}^{(n)}$ is empty). Let us remark now that for any sequence $z_{1}, z_{2}, \ldots, z_{n}$ of elements of $A$ we have

$$
\left|\sum_{k=1}^{n} z_{k}\right|^{2} \leqslant n \sum_{k=1}^{n}\left|z_{k}\right|^{2}
$$

This easily follows by induction from the inequality $x^{*} y+y^{*} x \leqslant x^{*} x+y^{*} y$. Put

$$
B_{m}=\sum_{I}\left|\sum_{k \in I} y_{k}\right|^{2}
$$

where $I$ runs over all intervals which appear as the elements of the partitions of $\left(0,2^{m}\right]$. Then we have

$$
\left|t_{n}\right|^{2} \leqslant(m+1) \sum_{j=0}^{m}\left|\sum_{k \in I_{j}^{(n)}} y_{k}\right|^{2} \leqslant(m+1) B_{m} .
$$

Moreover, $B_{m}$ does not depend on $n \in\left(0,2^{m}\right]$ and(5) holds, which completes the proof of the proposition.

Proof of Theorem 2. Put $S_{N}=(1 / N) \sum_{k=1}^{N} x_{k}$. Let $2^{k}<N \leqslant 2^{k+1}$. Then

$$
\begin{aligned}
\left|S_{N}-S_{2^{k}}\right|^{2} & =\left|\left(\frac{1}{N}-\frac{1}{2^{k}}\right) \sum_{s=1}^{2^{k}} x_{s}+\frac{1}{N} \sum_{s=2^{k}+1}^{N} x_{s}\right|^{2} \\
& \leqslant 2\left[\left(\frac{1}{N}-\frac{1}{2^{k}}\right)^{2}\left|\sum_{s=1}^{2^{k}} x_{s}\right|^{2}+\frac{1}{N^{2}}\left|\sum_{s=2^{k}+1}^{N} x_{s}\right|^{2}\right] .
\end{aligned}
$$


Applying the Proposition, we have that

$$
\left|S_{n}-S_{2^{k}}\right|^{2} \leqslant 2^{1-2 k}\left[\left|\sum_{s=1}^{2^{k}} x_{s}\right|^{2}+(k+2) B_{k}\right]
$$

where $B_{k}$ is a positive operator, independent on $N \in\left(2^{k}, 2^{k+1}\right]$ and such that

$$
\phi\left(B_{k}\right) \leqslant(k+2) \sum_{s=2^{k}+1}^{2^{k+1}} \phi\left(\left|x_{s}\right|^{2}\right)
$$

thus, for $2^{k}<N \leqslant 2^{k+1}$, we have

$$
\left|S_{N}-S_{2^{k}}\right|^{2} \leqslant D_{k}
$$

where $D_{k} \in A_{+}$, and

$$
\phi\left(D_{k}\right) \leqslant 2^{1-2 k}\left[\sum_{s=1}^{2^{k}} \phi\left(\left|x_{s}\right|^{2}\right)+(k+2) \sum_{s=2^{k}+1}^{2^{k+1}} \phi\left(\left|x_{s}\right|^{2}\right)\right] .
$$

By the assumptions of the theorem, we have

$$
\begin{aligned}
\sum_{k=1}^{\infty} \phi\left(D_{k}\right) \leqslant & \sum_{k=1}^{\infty} 2^{1-2 k} \frac{2^{2 k}}{k^{2}} \sum_{s=1}^{2^{k}} \phi\left(\left|x_{s}\right|^{2}\right)\left(\frac{\log s}{s}\right)^{2} \\
& +\sum_{k=1}^{\infty} 2^{1-2 k} \frac{2^{2 k}}{k^{2}}(k+2)^{2} \sum_{s=2^{k}+1}^{2^{k+1}} \phi\left(\left|x_{s}\right|^{2}\right)\left(\frac{\log s}{s}\right)^{2} \\
\leqslant & \sum_{s=1}^{\infty} \phi\left(\left|x_{s}\right|^{2}\right)\left(\frac{\log s}{s}\right)^{2}\left(\sum_{k=1}^{\infty} \frac{2}{k^{2}}+\text { const. }\right)
\end{aligned}
$$

Moreover,

$$
\begin{aligned}
\sum_{k=1}^{\infty} \phi\left(\left|s_{2^{k}}\right|^{2}\right) & =\sum_{k=1}^{\infty} \frac{1}{2^{2 k}} \sum_{s=1}^{2^{k}} \phi\left(\left|x_{s}\right|^{2}\right) \\
& \leqslant \sum_{k=1}^{\infty} 2^{-2 k} \frac{2^{2 k}}{k^{2}} \sum_{s=1}^{\infty} \phi\left(\left|x_{s}\right|^{2}\right)\left(\frac{\log s}{s}\right)^{2}<\infty
\end{aligned}
$$

Let $\varepsilon>0$ be given. By (15) and (16) one can find a sequence $\left(\varepsilon_{k}\right)$ of positive numbers such that $\varepsilon_{k} \rightarrow 0$ and

$$
\sum_{k=1}^{\infty} \varepsilon_{k}^{-1} \phi\left(\left|S_{2^{k}}\right|^{2}+D_{k}\right)<\frac{\varepsilon}{2} .
$$

By Theorem 1, there is a projection $p \in A$ with $\phi(p) \geqslant 1-\varepsilon$ and such that

$$
\left\|p\left|S_{2^{k}}\right|^{2} p\right\|<2 \varepsilon_{k} \text { and }\left\|p D_{k} p\right\|<2 \varepsilon_{k} .
$$

Thus, for $2^{k}<N \leqslant 2^{k+1}$, we have the estimation

$$
\begin{aligned}
\left\|S_{N} p\right\|^{2} & =\left\|\left(S_{N}-S_{2^{k}}\right) p+S_{2^{k}} p\right\|^{2} \\
& \leqslant 2\left[\left\|\left(S_{n}-S_{2^{k}}\right) p\right\|^{2}+\left\|S_{2^{k}} p\right\|^{2}\right] \\
& =2\left[\left\|p\left|S_{N}-S_{2^{k}}\right|^{2} p\right\|+\left\|p\left|S_{2^{k}}\right|^{2} p\right\|\right] \\
& \leqslant 2\left[\left\|p D_{k} p\right\|+\left\|p\left|S_{2^{k}}\right|^{2} p\right\|\right]<8 \varepsilon_{k} \rightarrow 0 \quad \text { as } N \rightarrow \infty .
\end{aligned}
$$


This means that $S_{N}=(1 / N) \sum_{s=1}^{N} x_{s}$ converges to zero almost uniformly, which ends the proof.

3. Comments. Let us compare Theorem 2 with the classical results. The Rademacher-Menchoff theorem on the almost sure convergence of orthogonal series [2] gives, via Kronecker's lemma, the following strong law of large numbers.

If, for a sequence $\left(X_{n}\right)$ of uncorrelated random variables,

$$
\sum_{n}\left(\frac{\log n}{n}\right)^{2} \operatorname{var}\left(X_{n}\right)<\infty,
$$

then $(1 / n) \sum_{k=1}^{n}\left(X_{k}-E X_{k}\right) \rightarrow 0$ with probability one.

Of course, Theorem 2 can (and should) be treated as the noncommutative extension of the strong law of large numbers just formulated.

Under some conditions stronger than in Theorem 2 one can obtain better convergence of the averages. Namely, it is easy to prove the following two theorems.

THEOREM 3. Let $\left(x_{n}\right)$ be a sequence in $A$ orthogonal relative to a state $\phi$. If

$$
\sum_{s=1}^{\infty} a_{s} \phi\left(\left|x_{s}\right|^{2}\right)<\infty \quad \text { when } 0<a_{s} \downarrow 0,
$$

and

$$
\sum_{s=1}^{\infty} \frac{1}{s^{2} a_{s}}<\infty
$$

then $(1 / n) \sum_{s=1}^{n} x_{s} \rightarrow 0$ almost completely.

Proof. Put $s_{N}=N^{-1} \sum_{s=1}^{N} x_{s}$. Then

$$
\phi\left(\left|S_{N}\right|^{2}\right)=\frac{1}{N^{2}} \sum_{s=1}^{N} \phi\left(\left|x_{s}\right|^{2}\right) \leqslant \frac{1}{N^{2} a_{N}} \sum_{s=1}^{N} a_{s} \phi\left(\left|x_{s}\right|^{2}\right),
$$

thus $\sum \phi\left(\left|S_{N}\right|^{2}\right)<\infty$. For $\varepsilon>0$, let us put $q_{N}=e_{\left[0, \varepsilon^{2}\right]}\left(\left|S_{N}\right|^{2}\right)$. Then $\left\|S_{N} q_{N}\right\|<\varepsilon$ for $N=1,2, \ldots$. Moreover,

$$
\sum_{N=1}^{\infty} \phi\left(q_{N}^{\perp}\right) \leqslant \varepsilon^{-2} \sum_{k=1}^{\infty} \phi\left(\left|S_{k}\right|^{2}\right)<\infty,
$$

which ends the proof.

The next theorem is a stronger version of Batty's result [3, Theorem 4.1].

THEOREM 4. Let $\left(X_{n}\right)$ be a uniformly bounded weakly independent (in the sense of [3]) sequence in $A$, with $\phi\left(X_{k}\right)=0$. Then $(1 / n) \sum_{s=1}^{n} X_{s} \rightarrow 0$ almost completely.

Proof. Let $\varepsilon>0$. Put $S_{N}=(1 / N) \sum_{s=1}^{N} X_{s}$. It is easy to show that $\phi\left(\left|S_{N}\right|^{4}\right) \leqslant$ $N^{-4}\left(3 N^{2}-N\right)$, hence $\sum_{N} \phi\left(\left|S_{N}\right|^{4}\right)<\infty$. Put $q_{N}=e_{\left[0, \varepsilon^{4}\right)}\left(\left|S_{N}\right|^{4}\right)$. It follows that $\sum_{N} \phi\left(q_{N}^{\perp}\right)<\infty$ and $\left\|S_{n} q_{N}\right\|^{4} \leqslant\left\|\left|S_{N}\right|^{4} q_{N}\right\|<\varepsilon^{4}$ for $N=1,2, \ldots$, which ends the proof.

4. Appendix. In this section we discuss the relationship between the almost uniform and almost complete convergence. Let us remark first that if $\phi$ is a trace, then the almost complete convergence implies the almost uniform convergence. 
Indeed, let $X_{n} \rightarrow 0$ almost completely. Then there is a sequence of projections $q_{n}$ such that $\left\|X_{n} q_{n}\right\|<\varepsilon$ for $n=1,2, \ldots$, and $\sum_{n} \phi\left(q_{n}^{\perp}\right)<\infty$. Putting $p_{n}=\Lambda_{s=n}^{\infty} q_{s}$, we have $\phi\left(1-p_{n}\right) \leqslant \sum_{s=n}^{\infty} \phi\left(1-q_{s}\right) \rightarrow 0$. The last condition implies the almost uniform convergence [9, Theorem 3.1].

When $\phi$ is a state, we have the following

TheORem 5. Let $A$ be a von Neumann algebra with a faithful normal state $\phi$. Let $\left(X_{n}\right)$ be a bounded sequence in $A$. If $X_{n} \rightarrow X$ almost completely, then $X_{n} \rightarrow X$ almost uniformly.

Proof. We can assume that $\left\|X_{n}\right\| \leqslant 1$ and $x=0$. Let $\varepsilon>0$. We find a sequence $\left(q_{n}\right)$ of projections in $A$ such that $\sum_{n} \phi\left(q_{n}^{\perp}\right)<\infty$ and $\left\|X_{n} q_{n}\right\|<\varepsilon$ for $n=1,2, \ldots$ Let us fix a sequence $\left(\varepsilon_{n}\right)$ of positive numbers such that $\varepsilon_{n} \rightarrow 0$ and $\sum_{n=1}^{\infty} \phi\left(1-q_{n}\right) \varepsilon_{n}^{-1}<$ $\varepsilon / 2$. By Theorem 1 there is a projection $p \in A$ with $\phi\left(p^{\perp}\right)<\varepsilon$ and such that $\left\|p q_{n}^{\perp} p\right\|=\left\|q_{n}^{\perp} p\right\|^{2}<2 \varepsilon_{n}$ for $n=1,2, \ldots$ Then we have

$$
\begin{aligned}
\left\|X_{n} p\right\| & \leqslant\left\|X_{n} q_{n} p\right\|+\left\|X_{n} q_{n}^{\perp} p\right\| \leqslant\left\|X_{n} q_{n}\right\|+\left\|q_{n}^{\perp} p\right\| \\
& \leqslant \varepsilon+\left(2 \varepsilon_{n}\right)^{1 / 2}<2 \varepsilon \text { for } n>M_{0}(\varepsilon) .
\end{aligned}
$$

Thus the following condition is satisfied:

For each $\varepsilon>0$ there is a projection $p$ with $\phi(p) \geqslant 1-\varepsilon$ and such that $\left\|X_{n} p\right\|<\varepsilon$ for $n>n_{0}(\varepsilon)$.

We shall show that the condition (*) implies the almost uniform convergence of $x_{n}$ to zero. We first prove that $(*)$ implies:

For each $\varepsilon \geqslant 0$ and for each projection $q \in A$ there is a (**) projection $r \in A$ such that $r \leqslant q, \phi(q-r)<\varepsilon$ and $\left\|X_{n} r\right\|<\varepsilon$ for $n$ large enough.

Indeed, let $0<\varepsilon_{n} \rightarrow 0$. By (*) we find in $A$ a sequence of projections $r_{n}$ with $\phi\left(r_{n}\right)>1-\varepsilon_{n}$, and a sequence of positive integers $m(n)$ such that $\left\|X_{m} r_{n}\right\|<\varepsilon_{n}$ for $m>m(n)$. Let a projection $q \in A$ be given. Then $\phi\left(q r_{n}^{\perp} q\right) \rightarrow 0$ and we can fix $n_{0}$ such that $\varepsilon_{n_{0}}<\varepsilon$ and $\phi\left(q r_{n_{0}}^{\perp} q\right)<\varepsilon^{4}$. Let $r=q e_{\left[0 \cdot \varepsilon^{2}\right]}\left(q r_{n_{0}}^{\perp} q\right)$. Then we have $r \leqslant q$, $\phi(q-r)<\varepsilon^{2}$ and $\left\|r_{n_{0}}^{\perp} r\right\|<\varepsilon$. Moreover, $\left\|X_{m} r_{n_{0}}\right\|<\varepsilon_{n_{0}}$ for $m>m\left(n_{0}\right)$. Thus $\left\|X_{m} r\right\|$ $\leqslant\left\|X_{m} r_{n_{0}}^{\perp} r\right\|+\left\|X_{m} r_{n_{0}} r\right\|<2 \varepsilon$ for $m>m\left(n_{0}\right)$, which ends the proof of the implication $(*) \rightarrow(* *)$.

Let $\varepsilon>0$ be given. By $(* *)$ we can find in $A$ a sequence of projections $\left(p_{n}\right)$ such that $1=p_{0} \geqslant p_{1} \geqslant p_{2} \geqslant \cdots, \phi\left(p_{n}-p_{n+1}\right)<2^{-n} \varepsilon$ and $\left\|X_{m} p_{n}\right\|<2^{-n} \varepsilon$ for $m>$ $m\left(n_{0}\right)$. Put $p=\Lambda_{k} p_{k}$. Then $\phi\left(p^{\perp}\right)=\sum_{n} \phi\left(p_{n}-p_{n+1}\right)<\varepsilon$. Moreover, $\left\|X_{m} p\right\| \leqslant$ $\left\|X_{m} p_{n_{0}}\right\|<2^{-n_{0} \varepsilon}$ for $m>m\left(n_{0}\right)$. That means that $X_{m} \rightarrow 0$ almost uniformly. The proof is completed.

\section{REFERENCES}

1. L. Acardi, A. Frigerio and J. T. Lewis, Quantum stochastic processes, Comm. Dublin Inst. Adv. Stud. Ser. A 29 (1980).

2. G. Alexits, Convergence problems of orthogonal series, Pergamon Press, New York, Oxford and Paris, 1961.

3. C. J. K. Batty, The strong law of large numbers for states and traces of a $W^{*}$-algebra, Z. Wahrsch. Verw. Gebiete 48 (1979), 117-191. 
4. N. Dang-Ngoc, Pointwise convergence of martingales in von Neumann algebras, Israel J. Math. 34 (1979), 273-280.

5. M. S. Goldstein, Theorems on almost everywhere convergence in von Neumann algebras, J. Operator Theory 6 (1981), 233-311. (Russian)

6. B. Kümmerer, $A$ non-commutative individual ergodic theorem, Invent. Math. 46 (1978), 139-145.

7. E. C. Lance, Ergodic theorems for convex sets and operator algebras, Invent. Math. 37 (1976), $201-214$.

8. A. Luczak, Some limit theorems in von Neumann algebras, preprint.

9. A. R. Padmanabhan, Convergence in measure and related results in finite rings of operators, Trans. Amer. Math. Soc. 128 (1967), 359-378.

10. M. Plancherel, Sur la convergence des series de fonctions orthogonal, C. R. Acad. Sci. Paris 157 (1913), 270-278.

11. M. Takesaki, Theory of operator algebras. I, Springer-Verlag, Berlin, Heidelberg and New York, 1979.

Department of Mathematics, University of Tennessee, KNoxville, Tennessee 37996 - 1300

Current address: Institute of Mathematics, Lodz University, Banacha 22, 90-456 Lodz, Poland 